Results In vitro polarisation with IL-10 specifically induced the expression of CD163 on macrophages, mimicking the phenotype in SpA synovitis. IL-10 and IL-4 polarised macrophages secreted less TNF than classically, IFN- $\gamma$-polarised macrophages $(p<0.05)$. In contrast, IL-4 polarised macrophages expressed more tmTNF compared to the IFN- $\gamma$ polarised macrophages $(p<0.05)$, with a similar trend for IL-10 polarised macrophages. In line with these in vitro data, the sTNF SF levels were significantly lower in SpA compared to RA $(p=0.01)$ despite similar TNF mRNA levels in ST. This was not related to higher expression of TNF receptors as both TNFR1 and TNFR2 were similarly expressed in ST, both at protein and mRNA levels. On the contrary, the SF levels of sTNFR1 and sTNFR2, which are both cleaved from the cell membrane by the same enzyme as tmTNF, were even decreased in SpA versus RA. Investigating the potential pathophysiological role of relative overexpression of tmTNF versus sTNF in vivo, clinical analysis revealed that tmTNF transgenic mice developed arthritis, resulting in deformation and loss of grip strength, and spondylitis as evidenced by crinkled tails with a $100 \%$ incidence. Axial and peripheral joint inflammation was confirmed by histology. In contrast to mouse strains overexpressing sTNF, tmTNF tg mice did not develop systemic disease and weight loss and showed clear signs of osteoproliferation on histology.

Conclusions tmTNF is relatively overexpressed by CD163+ alternatively polarised macrophages in SpA synovitis and leads to an axial and peripheral SpA phenotype in transgenic mice.

\section{A2.16 SYNOVIAL FLUID NEUTROPHILS UNDERGOING NETOSIS CONTRIBUTE TO JOINT INFLAMMATION BY PRODUCING CITRULLINATED AUTOANTIGENS}

doi:10.1136/annrheumdis-2013-203215.16

${ }^{1}$ Julia Spengler, 'Bozo Lugonja, ${ }^{2}$ Andrew Creese, ${ }^{3}$ Anthony Nicholas, ${ }^{2}$ Mike Milward, 'Mark Pearson, ${ }^{1}$ Christopher D Buckley, ${ }^{1}$ Andrew Filer, ${ }^{1}$ Karim Raza, ${ }^{2}$ Paul R Cooper, ${ }^{2}$ lain LC Chapple, 'Dagmar Scheel-Toellner. 'Centre for Translational Inflammation Research, School of Immunity \& Infection, College of Medical \& Dental Sciences, University of Birmingham Research Laboratories, Queen Elizabeth Hospital, Birmingham B15 2WD; 'University of Birmingham, School of Dentistry, St. Chads Queensway, Birmingham B4 6NN, UK; '3niversity of Alabama at Birmingham, Birmingham AL 35294, USA.

Objectives Anti-citrullinated protein antibodies (ACPA) are characteristically detected in patients with rheumatoid arthritis (RA) and growing evidence suggests that they are involved in disease pathogenesis. During disease flares large numbers of neutrophils enter the joint space of RA patients. These cells can extrude genomic DNA in an active process termed NETosis. NETosis critically depends on histone citrullination by peptidyl deiminase 4 (PAD4). Here we tested the hypothesis that activation and release of PAD4 during NETosis contributes to the production of autoantigens in the inflamed joint.

Materials and Methods Levels of extracellular DNA in the synovial fluid $(\mathrm{SF})$ of patients with RA $(\mathrm{n}=23)$ and osteoarthritis $(\mathrm{OA})$ $(n=15)$ or crystal arthritis $(n=6)$ were quantified using the non cell-permeable DNA dye SYTOX Green. PAD4, neutrophil elastase, citrullinated proteins and bound human IgG were labelled in association with DNA on smear preparations of RA SF and detected by immunofluorescence. NETs from SF and in vitro stimulated neutrophils were isolated and western blotting and mass spectrometry were used to identify proteins released during NET formation and proteins co-fractionated with NETs. PAD enzymatic activity was determined after NETosis in vitro and in the SF of patients with RA $(\mathrm{n}=9)$ and $O A(\mathrm{n}=9)$.

Results Extracellular DNA was detected in SF from patients with RA and crystal arthritis at significantly higher levels than in OA SF (RA versus OA $p<0.0001$ ). Association of neutrophil elastase with decondensed DNA both on smear preparations and in NETs isolated from RA SF suggests that the extracellular DNA is derived from
NETosis. On smear preparations PAD4 co-localised with decondensed chromatin in the SF of RA patients. PAD2 and PAD4 are released into the supernatant of in vitro stimulated neutrophils and were detected in NET fractions isolated from RA SFs. PAD enzymatic activity was released from neutrophils during in vitro activated NETosis while PAD activity in SF from RA patients was significantly higher than in OA patients $(p<0.001)$. The products of PAD activity, citrullinated proteins, were released into the supernatant and co-fractionated with extracellular DNA during in vitro NETosis. Furthermore, citrullinated proteins and bound human immune complexes were detected on NETs isolated from RA SF.

Conclusions These findings suggest that neutrophil NETosis, as a source of extracellular activated PAD enzymes, is involved in the production of auto-antigens in the joints of RA patients. In ACPA positive patients the resulting immune complexes can contribute to the perpetuation of the inflammatory response.

\section{A2.17 SYNOVIOCYTES CHANGE PHENOTYPE AND FUNCTION AFTER TREG-DEPLETION IN ARTHRITIC MICE}

doi:10.1136/annrheumdis-2013-203215.17

${ }^{1} \mathrm{M}$ Böttcher, ${ }^{1} \mathrm{I}$ Irmler, ${ }^{2} \mathrm{C}$ Wunrau, ${ }^{2} \mathrm{~A}$ Korb-Pap, ${ }^{3} \mathrm{G}$ Schett, ${ }^{2} \mathrm{~T}$ Pap, ${ }^{1} \mathrm{~T}$ Kamradt. ${ }^{1}$ University Hospital Jena, Institute of Immunology, Jena, Germany; ${ }^{2}$ University Hospital Muenster, Institute of Experimental Musculoskeletal Medicine, Muenster, Germany; ${ }^{3}$ University Hospital Erlangen, Department of Internal Medicine-3 and Institute for Clinical Immunology, Erlangen, Germany

Purpose/Objective Immunizstion with Glucose-6-phosphate isomerase (G6PI) induces arthritis in susceptible strains of mice. Depletion of regulatory $\mathrm{T}$ cells (Tregs) prior to immunisation switches the usually acute, self-limiting course to a non-remitting, destructive arthritis. This provides a possibility to study molecular switches for the transition from acute, self-limiting to chronic, destructive arthritis within one mouse model.

To examine the role of fibroblast-like synoviocytes (FLS), which are known to modulate immune responses via the production of pro- and anti-inflammatory mediators, the phenotype and function of FLS from mice with either acute, self-limiting or non-remitting, destructive arthritis was studied.

Materials and Methods FLS from DBA/ 1 mice that developed either the acute or the chronic form of arthritis were isolated from joints over a time course of 56 days. To investigate the phenotype of FLS ELISA studies as well as zymography have been performed. For the functional clarification of those cells the matrix-associated transepithelial resistance invasion (MATRIN) assay and a cartilage attachment assay have been used. Furthermore, FLS have been transferred in vivo into the knee joints of immunodeficient mice and the joints have been scored histologically.

Results FLS from Treg-depleted mice produced significantly more cytokines (e.g. Interleukin 6 (IL-6)) upon stimulation with other cytokines, growth factors and TLR ligands. This increased susceptibility to cytokine stimulation in chronic animals compared to acute ones is observable throughout the disease course (56 days). Furthermore, the secretion and activity of matrix metalloproteases (MMPs) was enhanced in the FLS from chronic mice compared to samples from acute ones. Additional functional differences include the collagen-destructive potential and the potential to attach and eventually invade wild type cartilage. Here, FLS from Treg-depleted chronic arthritic mice showed a higher invasive and destructive potential Ultimately, FLS from Treg-depleted mice were able to destroy cartilage in immunodeficient mice.

Conclusions Our results are compatible with the hypothesis that uninhibited inflammation in the early phase of Treg-depleted mice causes the acquisition of an autonomously aggressive phenotype of synoviocytes which contribute to the switch from acute to chronic arthritis even in the absence of late support from $\mathrm{T}$ and B lymphocytes. 